\title{
Surgical Anatomy of the Liver
}

\section{Alex Emmanuel Elobu ${ }^{1}$, Vianney Kweyamba ${ }^{1}$, Rakesh Rai ${ }^{2}$}

${ }^{1}$ Fellow, Department of HPB Surgery and Organ Transplantation, Fortis Hospital Mulund, Mumbai, India

${ }^{2}$ Senior Consultant and Head of Department of HPB Surgery and Liver Transplantation, Fortis Hospital Mulund, Mumbai, India

\section{ABSTRACT}

The liver is the second largest human organ and has got a complex internal vascular and ductal anatomy. It is subdivided in segments and receives dual blood supply from the hepatic artery and portal vein. The hepatic veins drain the liver directly into the inferior vena to which the liver is intimately related. Anatomical variations are common. A thorough knowledge of the liver anatomy and its variations is of critical importance for safe and successful procedures and surgeries involving the liver.

Keywords: Liver Anatomy; Hepatic Anatomy; Surgical Anatomy; Portal Vein; Hepatic Vein; Hepatic Artery

\section{Overview}

The liver is the largest gland and second largest organ in the human body weighing up to 1500 gand receiving up to $25 \%$ of the cardiac output despite being only $2-3 \%$ of total body weight.

It is a wedgeshaped organ that lies protected within the rib cage in the right upper quadrant of the abdominal cavity and extends across the midline.

The liver is functionally and surgically divided into left and right hemilivers or lobes by an imaginary live drawn from the Inferior Vena Cava (IVC) posteriorly to the gallbladder fossa anteriorly. This line is called Cantlie's line and overlies the course of the middle hepatic vein. Additionally the right hemiliver is divided by the right hepatic vein into a right anterior and aposterior section. The left hemiliver on the other hand is divided into a left medial and a left lateral section by the left hepatic vein which is related to the umbilical fissure that contains the falciform ligament ${ }^{[1]}$. A transverse plane known as the portal plane at the level of bifurcation of the portal vein further subdivides each section into two segments as described by Couinaud ${ }^{[2]}$. The left lateral section is subdivided into Segments II superiorly and III inferiorly. Similarly, the left medial section form segments IVa superiorly and IVbinferiorly. On the hand, theright anterior section forms segment V and VIII while the right posterior formssegment VI and VII superiorly and inferiorly accordingly. The caudate lobe of the liver that lies between the IVC posteriorly and the portal vein bifurcation anteriorly makes segment $\mathrm{I}^{[3]}$.

\section{Blood supply}

Arterial supply of the liver contributes $20-25 \%$ of hepatic inflow and is from the common hepatic artery(CHA)which takes off together with the splenic and left gastric artery from the celiac trunk, a branch of the abdominal aorta. The CHA typically runs in the hepatoduodenal ligament andgives off the gastroduodenal (GDA) right gastric and supraduodenal arteries and continues as the hepatic artery proper (HAP). At the porta hepatis, the HAP divides in a Y shaped manner into a left and right branch to supply corresponding lobes of the liver. The cystic artery which supplies the gall bladder usually comes of the right hepatic artery ${ }^{[1]}$.

This arterial configuration is most common and occurs in up to $60-80 \%$ of the population ${ }^{[4,5]}$. A replaced right hepatic artery may originate from the Superior Mesenteric arteryin up to $10-21 \%$ of individuals while a replaced left gastric artery may arise from the left gastric artery in up to $3-10 \%$. Also, an accessory left hepatic and right hepatic artery may 
exist in up $1-8 \%$. Another variant to this anatomy is a CHA which may come off the SMA in about $1.5 \%$ of the population $^{[4,6,7]}$.

\section{Venous Supply}

The majority of the hepatic inflow (75-80\%) comes through the portal vein with nutrient rich but oxygen depleted blood form the gastrointestinal tract (via coronary vein, SMV and IMV) and the spleen and pancreas (via splenic vein) $)^{[1]}$. The portal vein $(\mathrm{PV})$ is formed by confluence of the splenic vein (having already received the inferior mesenteric vein)

and the superior mesenteric vein behind the neck of the pancreas. The PV then takes an oblique and superior course towards the liver in the hepatoduodenal ligament. It receives the left gastric vein a.k.a the coronary vein and a few other unnamed tributaries. It has a short intrahepatic course (about $2 \mathrm{~cm}$ ) before making a $\mathrm{T}$ shaped division into a left and right branch*. The right branchfurther divides into 2nd order anterior and posterior branches to supply the right anterior and posterior sections respectively. Third order branches will then supply individual liver segments within the respective sections The left portal vein has a curving caudad and anterior coursethat passes under segment IV to supply segmentsII, III and IV ${ }^{[8-11]}$. It then terminates in a connection to the ligamentum teres (obliterated foetal umbilical vein and may communicate to the IVC through the ligamentum venosus (obliterated foetal ductus venosus) ${ }^{[1]}$.

This portal anatomy is present in $65-80 \%$ of individuals. In up to $10-15 \%$ of individuals the portal vein trifurcates into right anterior, right posterior and left portal vein at a common place. Also, the right posterior portal vein may arise from the main portal vein instead of the right portal vein in $0.3-7 \%$ of individuals ${ }^{[9]}$.

\section{Venous drainage}

The blood from the liver is drained into the inferior vena cava (IVC) via the hepatic veins. Commonly three veins drain the liver with the segment I (caudate lobe) draining directly via one or more short veins into the retrohepatic IVC. The right hepatic vein is the largest of the three veins, and receives tributaries from both anterior and posterior sections of the right hemiliver before opening into the IVC after a short $1-2 \mathrm{~cm}$ extrahepatic course. The middle hepatic vein drains the right anterior and left medial sections while the left hepatic vein drains the left lateral section. The middle and left hepatic veinscommonly confluence and drain as one into the IVC after a short (up to $2 \mathrm{~cm}$ ) extrahepatic course. In upto $15 \%$ of individuals an accessory right hepatic vein may exist and also drain into the IVC ${ }^{[1]}$.

\section{Biliary system}

The biliary system is a confluence of tributaries starting from the liver lobules eventually forming into segmental ducts then section ducts which in turn form the left and right hepatic ducts. Segment I (caudate lobe) is commonly drained by several ducts into the right and left hepatic ducts. The left and right hepatic ducts confluence to form the common hepatic duct which after receiving the cystic duct from the gall bladder becomes the common bile duct which empties into the duodenum. The course of the intrahepatic ductal system follows the course of the portal venous branches ${ }^{[12]}$.

The hepatoduodenal ligament arises as the lateral extension of the left triangular ligament and forms the lower margin of the lesser omentum and anterior border of the foramen of Winslow. It contains the portal triad i.e. hepatic artery, portal vein and bile ducts running to porta hepatis with the ductal system anterior, the artery behind and to the left of the ducts and the portal vein below the artery. This configuration is maintained in the intrahepatic course and with each segment receiving branches of the portal triad ${ }^{[1]}$.

Knowledge of this internal vascular and segmental anatomy is key to successful and safe liver procedures including diagnostic and interventional procedures (e.g. biopsy, trans-arterial embolization, portosystemic shunting), pre and intraoperative imaging, anatomical and non-anatomical liver resections, trauma surgery, and liver transplantation.

\section{References}

1. Leslie H Blumgart, LH. S.a.R.P.D. Surgical and radiologic anatomy of the liver, biliary tract, and pancreas, in blumgart's surgery of the liver, biliary tract and pancreas, W.R. Jarnagin, Editor, Elsevier 2017; 2-59.

2. Couinaud C. Liver anatomy: Portal (and suprahepatic) or biliary segmentation. Dig Surg 1999; 16(6):459-67. 
3. The Brisbane 2000 Terminology of Liver Anatomy and Resections. HPB 2000; 2(3):333-339.

4. Noussios G, Dimitriou I, Chatzis I, et al. The main anatomic variations of the hepatic artery and their importance in surgical practice: review of the literature. J Clin Med Res 2017; 9(4): 248-52. DOI: 10.14740/jocmr2902w.

5. Arifuzzaman M, Nasim Naqvi SS, Adel H, et al. Anatomical variants of celiac trunk, hepatic and renal arteries in a population of developing country using multidetector computed tomography angiography. J Ayub Med Coll Abbottabad 2017; 29(3):450-454.

6. Shukla PJ, Barreto SG, Kulkarni A, et al. Vascular anomalies encountered during pancreatoduodenectomy: Do they influence outcomes? Ann Surg Oncol 2010; 17(1):186-93. DOI: 10.1245/s10434-009-0757-1.

7. Hiatt JR, Gabbay J, Busuttil RW. Surgical anatomy of the hepatic arteries in 1000 cases. Ann Surg 1994; 220(1): 50-2.
8. Najah H, Ammar H, Gupta R, et al. Segmental branching pattern of the left portal vein: Anatomical characteristics and clinical implications. Clin Anat 2017. DOI: $10.1002 /$ ca.23009.

9. Iqbal S, Iqbal R, Iqbal F. Surgical implications of portal vein variations and liver segmentations: A recent update. J Clin Diagn Res 2017; 11(2):p. Ae01-5. DOI: 10.7860/JCDR/2017/25028.9453.

10. Schmid S, Demartines N, Soler L, et al. Portal vein normal anatomy and variants: Implication for liver surgery and portal vein embolization. Semin Intervent Radiol 2008; 25(2):86-91. DOI: 10.1055/s-2008-1076688.

11. Cheng YF, Huang TL, Lee TY, et al. Variation of the intrahepatic portal vein; angiographic demonstration and application in living-related hepatic transplantation. Transplant Proc 1996; 28(3):1667-8.

12. Hund M, Bhimji SS. Anatomy, abdomen, biliary ducts, in statpearls. StatPearls Publishing LLC: Treasure Island (FL) 2018. 